\title{
4.6. Les potins du centre-ouest et du sud-ouest de la Gaule : état de la question
}

\section{Richard Boudet $\uparrow$}

\begin{abstract}
Résumé. Les potins sont particulièrement mal connus dans le centre-ouest et le sud-ouest de la Gaule. Seule la région de Toulouse semble en avoir émis. Pour le reste, il s'agit généralement d'espèces importées provenant du centre et de l'est de la Gaule. Cette étude permet donc de préciser les limites occidentales de la diffusion des potins gaulois.
\end{abstract}

Zusammenfassung. Die Kenntnisse über Potinmünzen aus Zentral/Ost- und Süd/West Galliens sind besonders lückenhaft. Toulouse scheint die einzige Produktionsgegend zu sein. Bei den übrigen Potins handelt es sich grösstenteils um aus 7entral- und Ost-Gallien importierte Münzen. Diese Studie erlaubt es, die westlichen Grenzen der Verbreilung der gallischen Potinmünzen zu präzisieren.

Les potins sont particulièrement mal connus dans le centre-ouest et le sud-ouest de la Gaule. Ils représentent un très faible pourcentage de la circulation monétaire régionale pour la fin de l'Âge du Fer. Il s'agit pour la plupart d'espèces importées provenant du centre et de l'est de la Gaule. Seule la région de Toulouse dispose d'une émission de diffusion très réduite dont le droit et le revers présentent une même sorte de T (LT X, 3419). Le site de Vieille-Toulouse a livré la série la plus abondante de ce monnayage (plusieurs dizaines d'exemplaires au bas mot) et pourrait par là même être le site émetteur. Malheureusement la très grande majorité des potins régionaux ne provient pas de contextes sûrement datés. La présente contribution se bornera donc à un simple inventaire de la documentation disponible et à un premier essai de classement.

Engager une étude des potins découverts dans le centre-ouest et le sud-ouest de la Gaule est à la fois chose aisée et chose délicate. Chose aisée car on dispose de plusieurs inventaires ou travaux régionaux (Hiernard, 1987 pour le centre-ouest; Boudet, 1987 pour la Girondc ct Depeyrot, 1985 pour une partie de Midi-Pyrénées) permettant de se faire assez rapidement une idée de la documentation disponible et de se rendre à l'évidence : les potins sont très peu nombreux. Chose délicate car ces espèces ont dans l'ensemble, jusqu'à une date récente, assez peu attiré l'attention des numismates : elles sont donc peu signalées. On est frappé à la lecture des publications consacrées aux grands ensembles numismatiques trouvés sur des sites de la fin de l'Âge du Fer et/ou occupés dans les premiers temps de l'époque gallo-romaine (Mouliets-et-Villemartin en Gironde, Montmaurin et
Vieille-Toulouse en Haute-Garonne) de la faiblesse de la représentation des potins ou de leur absence. L'ancienneté des découvertes ne fait rien à l'affaire. Une série constituée de manière récente comme celle de Mouliets-et-Villemartin ne connaît que deux potins sur un total de près de deux cent cinquante monnaies (Sireix et alii, 1983).

Deux nuances doivent cependant être apportées à ce premier constat pessimiste. Le centre-ouest est un peu mieux pourvu, comme on va le voir, en potins du fait de. sa plus grande proximité des centres émetteurs (Hiernard, 1987). D'autre part, il est très probable que le Toulousain ait connu une émission de potins portant sur les deux faces une sorte de $\mathrm{T}$ dont la diffusion est localement très limitée (LT X, 3419).

Il convient dans un premier temps de tenter un inventaire des monnaies connues (d'après Depeyrot, 1985 et diverses recherches effectuées par nos soins dans la documentation concernant le sud-ouest de la Gaule : aucun potin n'a cncorc ćtć signalć cn $\Lambda$ riège, dans le Gers, les Hautes-Pyrénées, les Landes, le Tarn-et-Garonne et les Pyrénées-Atlantiques).

Le classement qui suit est organisé par ordre alphabétique de départements constituant les régions actuelles de Poitou-Charentes, Aquitaine et Midi-Pyrénées. On donne ensuite le nom de la commune de découverte, le lieu-dit et la fonction ancienne probable du site, puis un inventaire des monnaies avec le type (le renvoi à La Tour, 1892 lorsqu'il est connu) et enfin l'étude de référence lorsque la monnaie est publiée (fig. 61). 


\section{POITOU-CHARENTES}

\section{VENDÉE}

1. NAL.LIERS, l'llot-les-Vases (agglomération)

- 9 potins « à la tête diabolique » ou assimilés (Hiernard 1987, p. 738).

- 2 potins sans autre précision (Hiernard, 1987, p. 738).

- 1 potin «à la grosse tête " des Séquanes LT XVI, 5390 (Hiernard, 1987, p. 1123, n 183 ).

\section{LE LANGON}

- "Bon nombre " de potins sans autre précision (Hiernard, 1987, p. 738).

\section{SAINTE-HERMINE}

- 1 potin assimilé «à la tête diabolique " (Hiernard, 1987, p. 738).

\section{DEUX-SÈVRES}

4. FAYE-L'ABBESSE, les Crânières (sanctuaire)

- 30 potins sans autre précision (Gendron, Gomez de Soto, 1986 , p. 90 ).

5. NIORT (agglomération)

- 8 potins " à la tête diabolique » (Hiernard, 1984, p. 77, $\mathrm{n}^{\circ} 44$ et p. 101, n 15,18 à 20$)$.

- 1 potin sans autre précision (Hiernard, 1987, p. 738).

6. LA CRÈCHE, la Fougeoire (agglomération?)

- 1 potin des Éduens BN 5273 (Hiernard, 1987, p. 1123, $\mathrm{n}^{\circ} 185$ )

7. PRAHECQ Saziré (agglomération?)

- 1 potin des Éduens BN 5267-5271 (Hiernard, 1987, p. 1123, $\left.\mathrm{n}^{\circ} 186\right)$.

\section{VIENNE}

8. VENDEUVRE-DU-POITOU, les Tours Mirandes (agglomération)

- 1 potin «à la grosse tête " des Séquanes LT XVI, 5401 (Hiernard, 1987, p. 1123, n¹84).

9. NAINTRÉ (agglomération)

- 7 potins «à la tête diabolique " (Hiernard, 1987, p. 738).

- 1 potin sans autre précision (Hiernard, 1987, p. 738).
10. INGRANDES (agglomération?)

- 1 potin « à la tête diabolique " (Hiernard, 1987, p. 738).

- 1 potin sans autre précision (Hiernard, 1987, p. 738).

11. POITIERS (agglomération)

- 12 potins au bas mot sans autre précision (Hiernard, 1987, p. 738).

- 1 potin de Gaule Belgique LT XXXVII, 9155 (Hiernard, 1987 , p. 1123, $\mathrm{n}^{\circ} 171$ ).

12. CHAUVIGNY, Saint-Pierre-les-Églises (agglomération)

- 1 potin des Éduens BN 4632 (Hiernard, 1987, p. 1123, $\mathrm{n}^{\circ} 187$ )

13. ANTIGNY, le Gué de Sciaux (agglomération)

- 26 et 36 potins « à la tête diabolique » et 47 assimilés (Hiernard, 1987, p. 738).

- 1 potin sans autre précision (Hiernard, 1987, p. 738).

- 1 potin des Carnutes BN 6160-6168 (Hiernard, 1987, p. 1123, $\left.{ }^{\circ} 175\right)$.

- 1 potin « au swastika » des pays de Loire BN 6292-6293 (Hiernard, 1987, p. 1124, n²13).

\section{Charente-Maritime}

14. MURON, la Couture (habitat)

- 8 potins " à la tête diabolique " ou assimilés : $4,47 \mathrm{~g}$, $3,57 \mathrm{~g}, 3,08 \mathrm{~g}, 3,04 \mathrm{~g}, 2,74 \mathrm{~g}, 2,68 \mathrm{~g}, 2,07 \mathrm{~g}$, et $1,51 \mathrm{~g}$ (Mainjonet, 1975, p. 55-56).

15. PONS (oppidum)

- 1 potin "à la tête diabolique "(Lassarade, 1986, p. 132).

\section{AQUITAINE}

\section{GIRONDE}

16. SOUI AC-SUR-MER, la Glaneuse

- 1 potin à « la tête diabolique » possible : 1,63 $\mathrm{g}$ (Boudet, Moreau, 1989, fig. 27, $n^{\circ} 8$ ).

17. BORDEAUX (agglomération)

- 1 potin « à la tête diabolique »: 3,05 g (Boudet, 1987, pl. $\left.27, n^{\circ} 9\right)$

-1 potin LT XVI, 5284 possible : 3,12 g (Boudet, 1987, p. 66). 


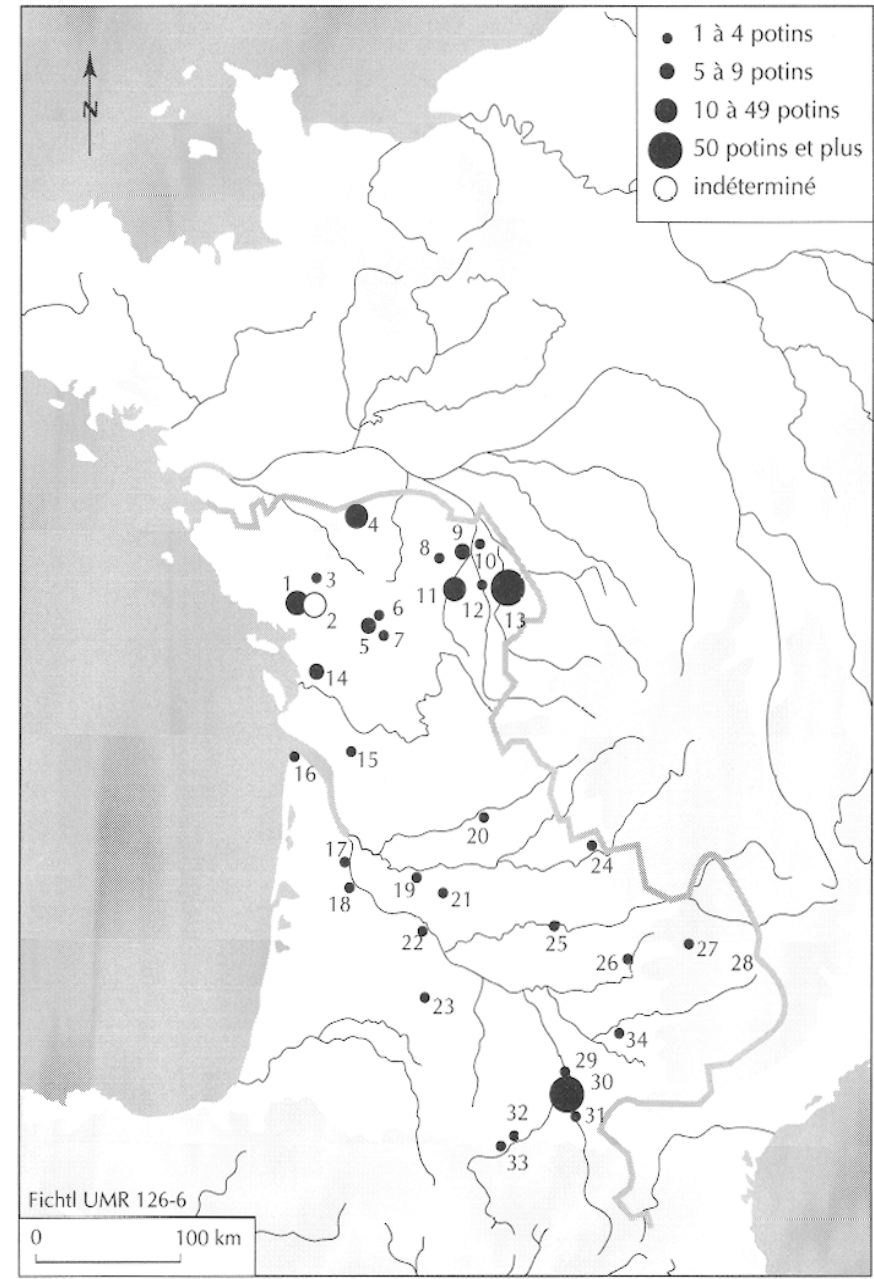

Fig. 61. Les potins découverts dans le centre-ouest et le sud-ouest de la France.

18. L'ISIE-SAINT-GEORGES, le Bourg (agglomération)

-1 potin « à la tête diabolique » (inédit : fouille R. Boudet).

- 1 potin illisible (inédit : fouille R. Boudet).

19. MOULIETS-ET-VILLEMARTIN, Lacoste (agglomération)

-2 potins « à la tête diabolique » : 2,54 g et 1,97 g (Boudet, 1987 , pl. $135, \mathrm{n}^{\circ} 232$ et 198$)$.

\section{DORDOGNE}

20. COULOUNIEIX-CHAMIERS, la Curade (oppidum)

- 1 potin des Sénons LT XXX, 7445 : 3,19 g (Chevillot, 1983 , p. 139).

\section{LOT-ET-GARONNE}

21. MOUSTIER, Sissac (bâtiment antique)

- 1 potin « au rameau " LT XXXXV, $8620: 4,32 \mathrm{~g}$ (inédit : renseignement J.-P. Noldin).

22. LE MAS-D'AGENAIS, Revenac (agglomération)

- 1 potin à " la grosse tête " LT XVI, 5401:3,60 g (Cadenat, 1982, p. 256, n85).

- 1 potin « à la tête diabolique » : $3 \mathrm{~g}$ (inédit : renseignement J.-P. Noldin).

23. SOS, Loustalet (agglomération)

- 1 potin «à la tête diabolique " (inédit : renseignement J.-P. Noldin).

\section{MIDI-PYRÉNÉES}

LOT

24. VAYRAC, le Puy d'Issolud (oppidum)

- 1 potin de l'est « à l'aigle " LT XVI, 5275 (Depeyrot, 1985 , p. 169).

- 1 potin à « la grosse tête " LT XVI, 5368 (Depeyrot, 1985, p. 169).

25. LUZECH, l'Impernal (oppidum)

- 1 potin à « la grosse tête " LT XVI, 5368 (Depeyrot, 1985, p. 165).

\section{AVEYRON}

26. SAVIGNAC 3 (agglomération)

- 1 potin «à la tête diabolique »: $3 \mathrm{~g}$ (Bénevent, Boudet, 1990, p. 45).

\section{RODEZ (agglomération)}

- 2 potins « à la tête diabolique " : 1,97 g (Depeyrot, 1982, p. 83, $\mathrm{n}^{\circ} 24$ ) et 2,74 g (Arramond, 1988, p. 92 , zone 1).

- 2 potins illisibles : $1,54 \mathrm{~g}$ (inédit : renseignement $\mathrm{L}$. Dausse; Arramond, 1988, p. 92, sondage 9).

28. SÉVÉRAC-LE-CHÂTEAU, Altès (villa)

- 1 potin des Carnutes BN 6151 : 3,40 g (inédit : renseignement P. Blanquet). 


\section{HAUTE-GARONNE}

29. TOUI.OUSE, Estarac (habitat?)

- 2 potins au « T » LT X, 3419 (Depeyrot, 1985, p. 87).

30. VIEILIE-TOULOUSE, la Planho (agglomération)

- 2 potins non identifiés (Savès, Villaronga, 1978, p. 41, $\left.\mathrm{n}^{\circ} 23-24\right)$.

- 3 potins BN 5660,5663 et 5677 «à la tête diabolique ": $3,16 \mathrm{~g}, 1,59 \mathrm{~g}$ et $3,28 \mathrm{~g}$ (Muret, Chabouillet, 1889, p. 126-127).

- 1 potin fruste : 3,25 g (Savès, Villaronga, 1979, p. 77, $\left.n^{\circ} 34\right)$.

- 2 potins " au swastika " des pays de Loire BN 6284 à $6294: 1,23 \mathrm{~g}$ (Savès, Villaronga, 1976, p. 185, $\mathrm{n}^{\circ} 35$ ) et 2,67 g (Savès, Villaronga, 1978, p. 41, n²2).

- 1 potin des Sénons LT XXX, $7465: 4,15$ g (Savès, Villaronga, 1975, p. 108, $\mathrm{n}^{\circ} 250$ ).

- 1 potin des Lingons IT XXXIII, 8329 : 3,33 g (Savès, Villaronga, 1976 , p. $\left.185, \mathrm{n}^{\circ} 38\right)$.

- 56 potins au « T » LT X, 3419 au bas mot (Depeyrot, 1985, p. 89 à 92).

- 1 potin «à la tête diabolique » (Depeyrot, 1985, p. 91, $\mathrm{n}^{\circ} 384$ ).

- 1 potin gaulois sans autre précision (Depeyrot, 1985, p. $91, \mathrm{n}^{\circ} 385$ ).

- 1 potin gaulois au quadrupède déformé (?) (Depeyrot, 1985, p. $\left.91, \mathrm{n}^{\circ} 469\right)$.

31. AUTERIVE, le Gazou (gué)

- 1 potin sans autre précision (Depeyrot, 1985, p. 64).

32. MARTRES-TOLOSANE, Chiragan (villa)

- 1 potin au « T » LT X, 3419 (Savès, 1976, p. 219).

33. SAINT-MARTORY, RN125, garage Duclos

- 1 potin «des Leuques » LT XXXVII, 9078 (Depeyrot, 1985, p. 81).

\section{TARN}

\section{MONTANS (agglomération)}

- 2 potins « au swastika » des pays de Loire BN 6292-6293 : 2,36 g et 2,22 g (Muret, Chabouillet, 1889, p. 139, $\mathrm{n}^{\circ} 6292$ et 6293$)$.

L'inventaire des potins connus dans le centre-ouest et le sud-ouest de la Gaule appelle un certain nombre de

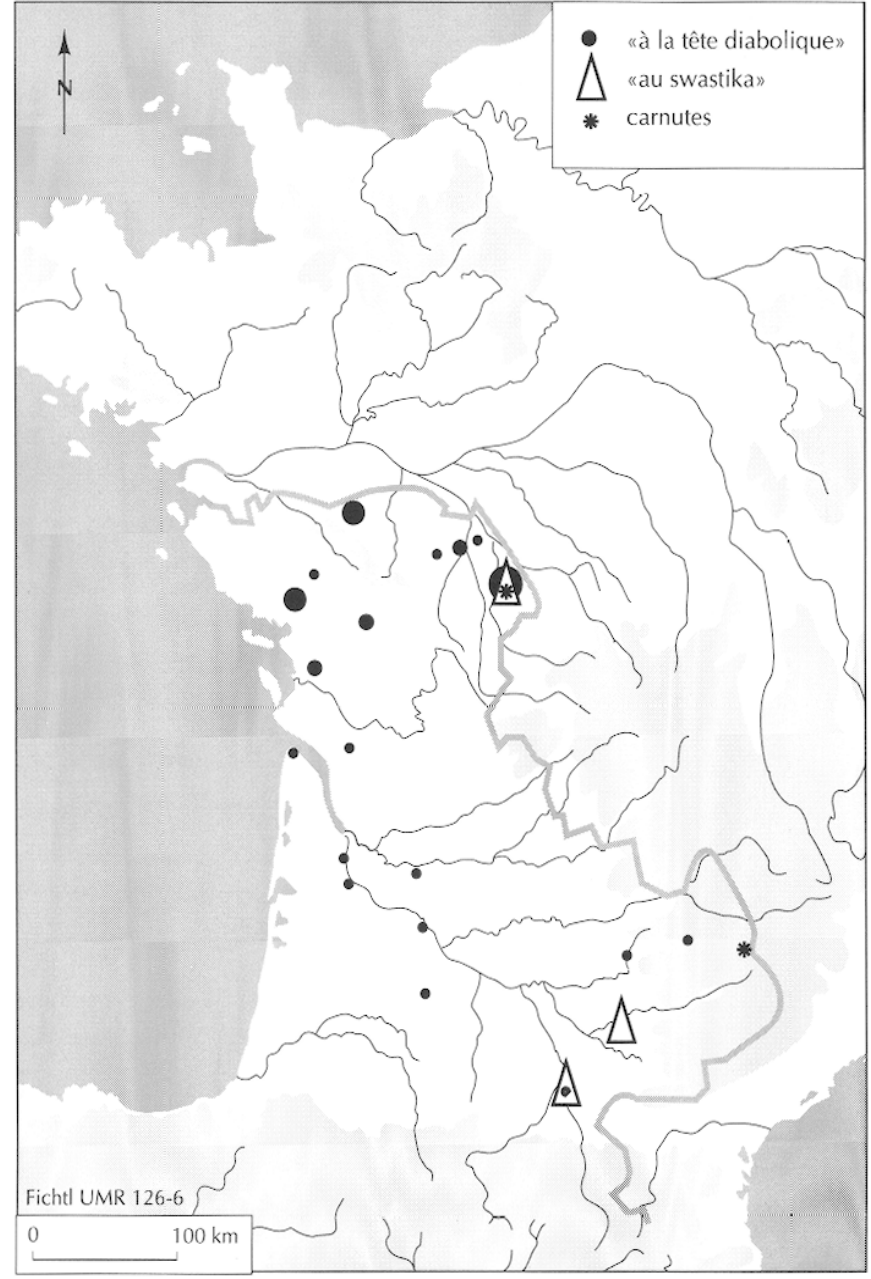

Fig. 62. Les potins originaires des pays de la Loire découverts dans le. centre-ouest et le sud-ouest de la France.

commentaires. Ce sont deux cent quatre-vingt-dix-sept potins qui ont été ici pris en considération : deux cents en centre-ouest sur quinze sites et quatre-vingt-dix-sept dans le sud-ouest sur dix-neuf sites. On notera dès maintenant le déséquilibre entre les deux régions sur lequel on reviendra. Cinquante-huit potins ne sont pas étudiables. Les deux cent trente-neuf autres potins peuvent ainsi se répartir :

- potins des pays de Loire : 165 dont

.158 « à la tête diabolique » sur 18 sites,

.5 « au swastika » sur 3 sites,

.2 carnutes sur 2 sites,

- potins de l'est de la France : 13 dont

.3 des Éduens sur 3 sites,

.2 des Sénons sur 2 sites,

.6 des Séquanes sur 6 sites,

.1 des Leuqucs sur 1 sitc, 


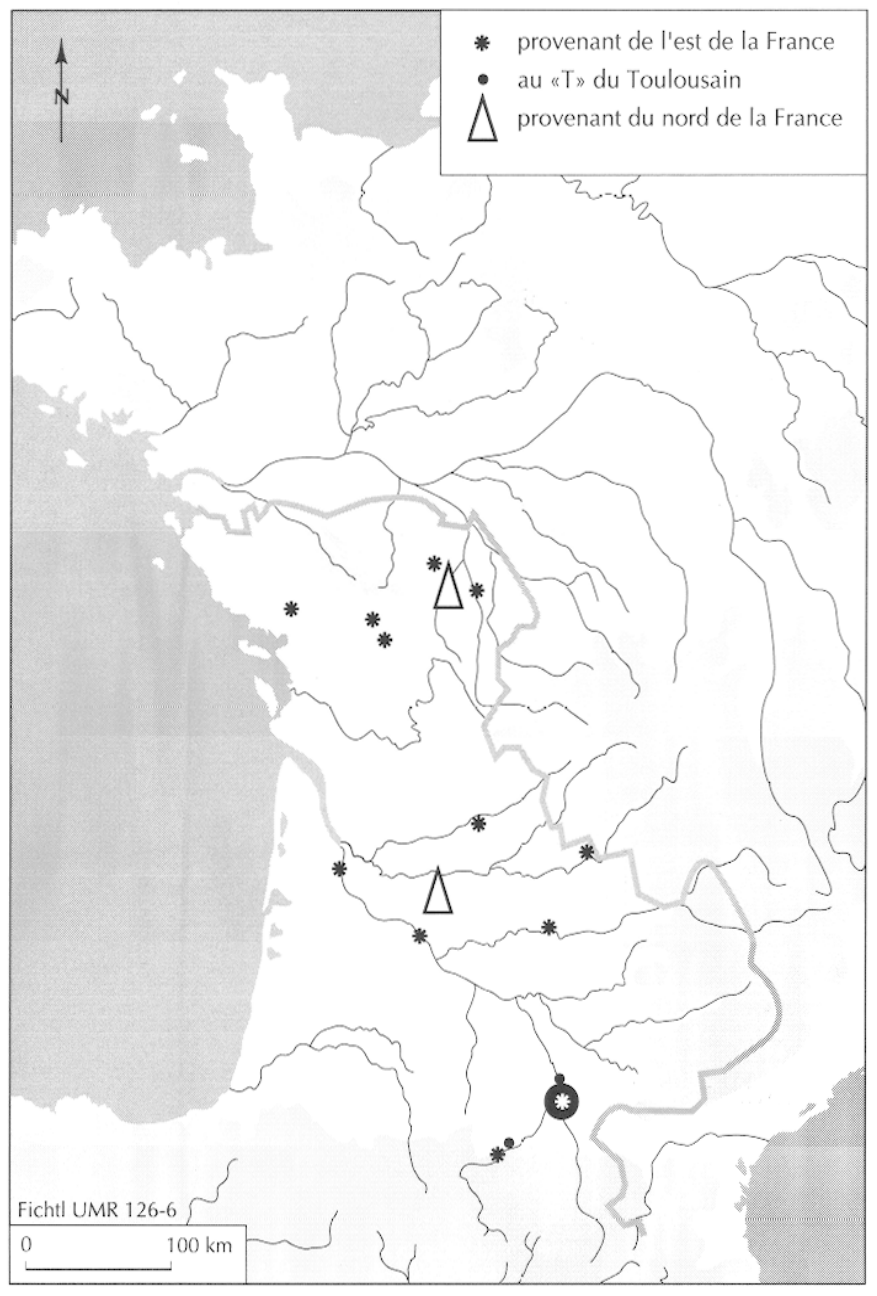

Fig. 63. Types divers de potins découverts dans le centre-ouest et le sud-ouest de la France.

. 1 des Lingons sur 1 site,

- potins du nord de la France : 2 dont

.1 « au rameau » sur 1 site,

. 1 LT XXXVII, 9155 sur 1 site,

- potins au « $\mathrm{T}$ » du Toulousain : 59 (au bas mot) sur 3 sites.

Les dix-huit départements répartis en trois régions administratives (Poitou-Charentes, Aquitaine et MidiPyrénées) qui ont fait l'objet de nos recherches ne connaissent actuellement que trente-quatre sites de découverte de potins. Les départements situés au sud de la Garonne n'en ont fourni aucun hormis Sos (Lot-etGaronne). L'alimentation régionale est essentiellement assurée par les pays de Loire avec une assez grande diffusion des potins « à la tête diabolique ", puis en quantités nettement plus faibles des potins " au swastika " et car- nutes (fig. 62). Les concentrations restent cependant localisées en Poitou-Charentes près des zones d'émission. Les autres types de potins signalés sont totalement exogènes et proviennent de l'est et du nord de la Gaule (fig. 63). Ils relèvent de l'anecdote. Il faut noter la concentration de ces espèces sur des sites à sanctuaires comme Nalliers, Faye-l'Abbesse ou Antigny.

Cette remarque en appelle une autre. L'émission et la concentration toulousaine du potin " au $\mathrm{T}$ " sont une curiosité. En effet, cette espèce ne circule pratiquement pas et ne se rencontre quasiment qu'à Vieille-Toulouse. Quelques exemplaires proviennent du puits XVI (Labrousse et alii, 1971) parmi des mobiliers attribuables au premier tiers du $\mathrm{I}^{\mathrm{er}} \mathrm{s}$. avant notre ère (Lequément, Vidal, 1986). Lne analyse métallographique de surface avait été réalisée pour le compte de G. Savès (Savès, 1976, p. $\left.89, \mathrm{n}^{\circ} 13 \mathrm{~B}\right)$ et a fourni les données suivantes :

$$
\begin{aligned}
& \mathrm{Cu}: 87,1 \% \\
& \mathrm{~Pb}: 2,5 \mathrm{~g} \% \\
& \mathrm{Sn}: 6, \lg \%
\end{aligned}
$$

L'étude de cette émission toulousaine reste à faire. On notera la présence sur le plateau de Vieille-Toulouse de deux sanctuaires reconnus : le premier datable de la première moitié du $\mathrm{I}^{\mathrm{er}} \mathrm{s}$. avant notre ère (inédit : renseignement $\mathrm{M}$. Vidal) et le second du milieu du même siècle. Dans plusieurs des puits découverts sur le site, on a pu mettre en évidence des zones de dépôt volontaire d'objets. La question doit maintenant se poser d'une probable vocation religieuse du plateau de Vieille-Toulouse à laquelle pourrait être associée l'émission des potins « au $\mathrm{T}$ ».

Au terme de cette contribution, on retiendra la faible diffusion en Gaule occidentale des potins principalement représentés par des espèces originaires des pays de Loire. Le potin toulousain " au $\mathrm{T}$ " pose le problème de leur fonction : espèces fiduciaires ou espèces à vocation religieuse? Régionalement, ces petites espèces (en admettant une vocation économique) pourraient avoir été remplacées par des frappes de petits bronzes romanisés portant les noms de dirigeants indigènes : CONTOVTOS, ATECTOTI(X), IVCCIOS, VRIDO RVF(VS), ANNICOIOS en Poitou-Charentes, Dordogne et moyenne vallée de Garonne (Hiernard, 1987), LVCTERIOS en Quercy ou TATINOS et ATTALLOS en RouergueAlbigeois (Boudet, 1990). Les espèces au nom 
d'ATTALLOS, mais aussi celles portant le nom de VIREDIOS en Poitou ont été frappées sur des flans en bronze obtenus comme les potins en chapelets ce qui a parfois eu pour effet de les assimiler à des potins. Il en va de même pour le bronze BN 4302-4303 du Berry présent dans la région ici considérée sur une dizaine de sites.

\section{Richard BOUDET}

\section{Bibliographie}

ARRAMOND J.-Ch.

1988, Un aspect de l'occupation humaine de Rodez antique, Cahiers d'Archéologie Aveyronnaise, 2, p. 83-97.

BÉNEVENT Chr., BOUdET R.

1990, Occupation du sol et circulation monétaire à la fin de l'Âge du fer dans l'ouest du département de l'Aveyron, Cahiers d'Arch. Aveyronnaise, 4, p. 36-50.

\section{BOUDET R.}

1987, L'Âge du Fer récent dans la partie méridionale de l'estuaire girondin ( $d u V^{t}$ au $I^{t r}$ siècle avant notre ère), Périgueux (Coll. Archéologies, 2).

1990, Numismatique et organisation du territoire du SudOuest de la Gaule à la fin de l'Âge du Fer : une première esquisse, in: Les Gaulois d'Armorique, La fin de l'Âge du Fer en Europe tempérée, Actes du XIIe colloque de l'AFEAF, Quimper, mai 1988, Rennes, p. 169-190 (3e suppl. à la $R A O$ ).

BOUDET R., MOREAU J.

1989, Numismatique antique du nord-Médoc (Gironde), Actes du XLI congrès d'Études régionales de la Fédération Historique du Sud-Ouest, Soulac-Pauillac-Saint-Germain-d'Esteuil (16 et 17 avril 1988), p. 105-133.

CADENat P.

1982, Nouvelles recherches dans la nécropole gallo-romaine d'Ussubium (dite aussi de Saint-Martin), commune du Masd'Agenais, Société Académique d'Agen.

\section{Chevillot Chr.}

1983, Résultats d'une coupe dans l'agger septentrional du Camp de César à la Curade, commune de CoulounieixChamiers (Dordogne), in :J. Collis, A. Duval et R. Périchon (éd.), Le Deuxième Âge du Fer en Auvergne et en Forez et ses relations avec les régions voisines, Saint-Étienne, p. 115144 (Centre d'Études Foréziennes).
DEPEYROT G.

1983, Les monnaies des fouilles de Rodez, Cahiers Numismatiques, p. 241.

1985, Les monnaies antiques des départements des Hautes-Pyrénées, de la Haute-Garonne, du Tarm-et-Garonne et du Lot, Sorèze.

GENDRON Chr., GOMEZ DE SOTO J.

1986, Le sanctuaire pré-romain de Faye-l'Abbesse (DeuxSèvres), Actes du VIII colloque de l'AFEAF, Bordeaux, p. 89-95 (1 ${ }^{\mathrm{er}}$ suppl. à Aquitania).

\section{HIERNARD J}

1984, Les monnaies antiques de Niort (Deux-Sèvres), Aquitania, 2, p. 59-104.

1987 : Les monnaies du Poitou antique, circulation monétaire et histoire, thèse de l'université de Bordeaux III (dactyl.).

LABROUSSE M., VIDAL M., MUII.ER A.

1971, Le puits funéraire XVI de Vieille-Toulouse, $96^{\circ}$ Congrès national des Sociétés Savantes, Toulouse, Archéologie, I, p. 63-95.

LASSARADE L.

1986, L'oppidum de Pons, Actes du VIII colloque de l'AFEAF, Bordeaux, p. 124-138 ( $1^{\mathrm{er}}$ suppl. à Aquitania).

LEQUÉMENT R., VIDAL M.

1986, Chronologie corrigée des oenochoés du type Kelheim trouvées dans les puits funéraires 8 et 9 de Toulouse, Estarac (Haute-Garonne), Mélanges offerts à M. Michel Labrousse, Pallas, hors série, p. 223-257.

MAINJONET M.

1975, Monnaies gauloises récoltées à Muron (CharenteMaritime), Revue de la Saintonge et de l'Aunis, 1, p. 49-56.

SAVÈs G.

1976, Les monnaies gauloises à la croix et assimilées du sud-ouest de la Gaule: Examen et catalogue, Toulouse.

SAVÈs G., VILLARONGA L.

1975, Les monnaies de la Péninsule ibérique trouvées en France dans la région Midi-Pyrénées, Acta Numismatica, V, p. 91-135.

1976, Acta Numismatica, VI, p. 107-130.

1977, Acta Numismatica, VI, p. 181-186.

1978, Acta Numismatica, VIII, p. 27-45.

1979, Acta Numismatica, IX, p. 63-84.

SireiX M., NoldiN J.-P., COlberT DE BEAUlieU J.-B., NONY D., RICHARD J.-Cl.

1983, Les monnaies de Mouliets-et-Villemartin (Gironde)

(1954-1982), Gallia, 41, p. 25-57. 


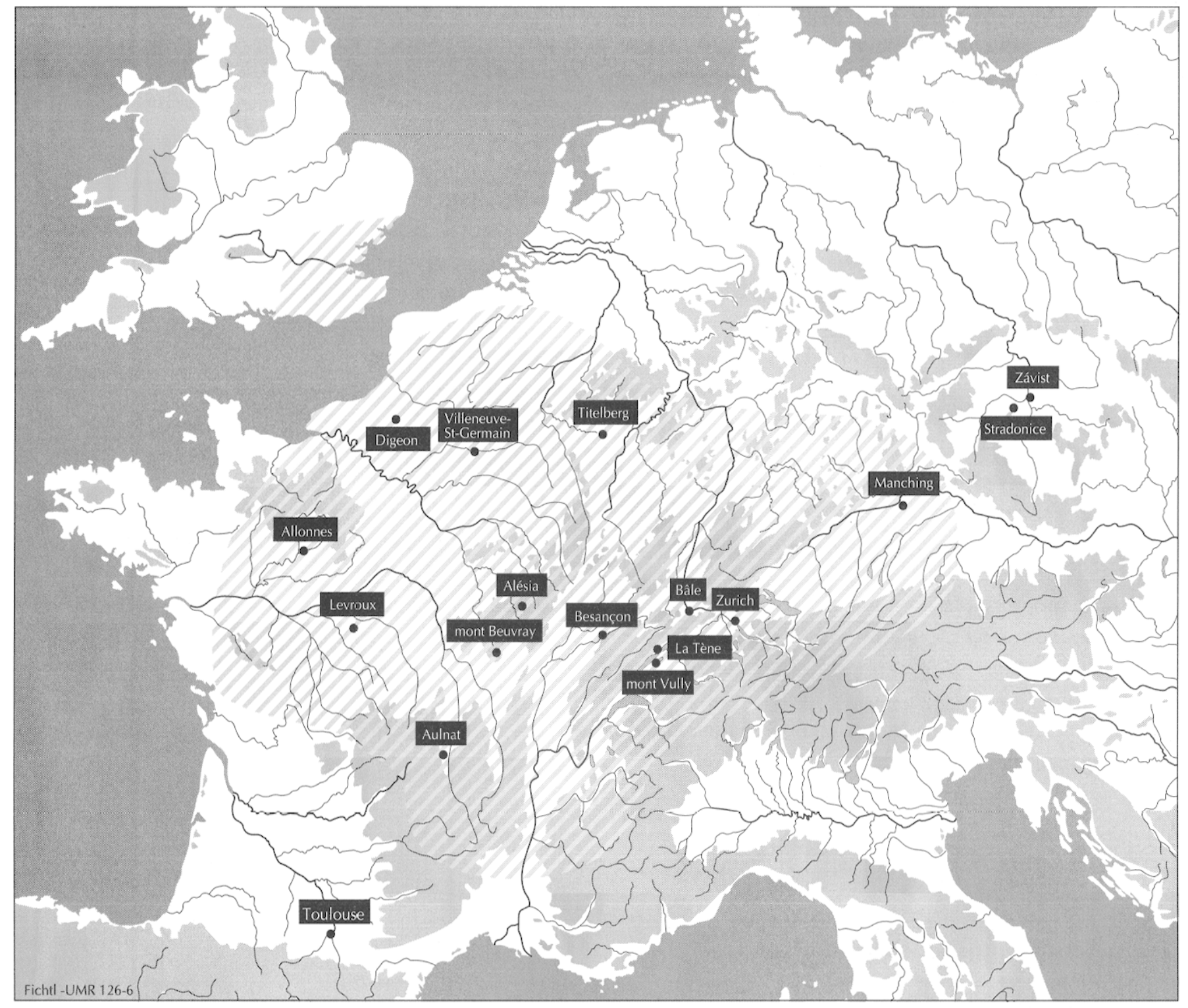

Fig. 64. Aire de diffusion générale des potins celtiques.

Ces six communications ont immédiatement mis en évidence deux phénomènes. D'une part, il existe deux types de potins : les uns ont une circulation limitée comme le type de Zurich tandis que d'autres, comme les potins " à la grosse tête " sont largement diffusés. D'autre part, les découvertes de potins restent négli- geables en Narbonnaise et sur l'ensemble de la façade atlantique de la Gaule, de même que dans le Bas-Rhin. On en trouve massivement dans le sud de l'Angleterre, le Nord et le Nord-Est, en Gaule Belgique, dans le Centre, l'Est y compris la Suisse occidentale et le HautRhin (fig. 64). 